\section{Synchronous Triple Primary Cancer of the Esophagus, Stomach and Jejunum: A Case Report and Review of Literature}

\author{
Wei-Peng Hu', Yu-Shang Yang', Jun-He Gou², Yong Yuan', \\ Wen-Ping Wang ${ }^{1}$ and Long-Qi Chen ${ }^{1 *}$
}

${ }^{1}$ Department of Thoracic Surgery, West China Hospital, Sichuan University, Chengdu, China

${ }^{2}$ Pathology department, West China Hospital, Sichuan University, Chengdu, China

\begin{abstract}
Synchronous multiple primary malignancies are relatively unusual. This report presents a 50-year-old male patient with durative dysphagia for over 1 year, who was diagnosed with advanced lower esophageal squamous carcinoma, gastric angle adenocarcinoma and Gastrointestinal Stromal Tumor (GIST) in the jejunum. Esophagectomy, total gastrectomy and segmental jejunectomy were performed via left thoracotomy and midline abdominal incision and systemic dissection of mediastinal and abdominal lymph nodes were also conducted during the operation. The remaining jejunum was used for esophageal substitution and a Roux-en-Y procedure was applied to restore the intestinal continuity. Fifteen days after the operation, the patient was discharged without any complication and received SOX chemotherapy for six cycles 3 months after surgery. After 12 months follow-up, the patient is alive without recurrence or metastasis and under regular follow-up. Therefore, we conclude that the surgical procedure and postoperative neo-adjuvant treatment are acceptable. To our knowledge, this is the first reported case of resected triple primary cancers involving the esophagus, stomach and jejunum synchronously.
\end{abstract}

Keywords: Esophageal cancer; Gastric cancer; GIST; Synchronous triple primary cancer

\section{Introduction}

Incidence of multiple primary tumors occurring in one patent is rare. It is the upper gastrointestinal tract, respiratory system, head and

*Corresponding author: Long-Qi Chen, Department of Thoracic Surgery, West China Hospital, Sichuan University, Chengdu, China, Tel: +86 2885422498; Fax: +86 2885422494; E-mail: drchenlq@gmail.com

Citation: Wei-Peng Hu, Yu-Shang Yang, Jun-He Gou, Yuan Y, Wen-Ping Wang, et al. (2016) Synchronous Triple Primary Cancer of the Esophagus, Stomach and Jejunum: A Case Report and Review of Literature. J Clin Stud Med Case Rep 3: 029

Received: November 26, 2015; Accepted: January 19, 2016; Published: February 03, 2016 neck region, or urogenital system that they appear more frequently, and the incidence ranges from $2 \%$ to $10 \%$ [1-6]. Moreover, synchronous multiple primary neoplasms are relatively rarer, whose incidence were only $1.2-1.9 \%$ of all primary cancers [7]. We report a patient with synchronous triple primary cancer (esophagus, stomach and jejunum), to our knowledge, which has never been reported.

\section{Case Presentation}

A 50-year-old man with progressive dysphagia for over 1 year was admitted to our hospital in September 2014. He was a heavy smoker for over 30 years, $>7$ packs per week. And he was also a heavy alcohol drinker for over 30 years. History of malignancy had never occurred in his family. There was no positive symptom detected after carful physical examination. The laboratory inspections, which contained the hemogram and biochemical examinations and carcinoembryonic antigen, did not reveal any abnormalities. Detection on chest X-ray and ECG revealed no anomalies. Pulmonary function test showed a mild damage. An approximately $3 \mathrm{~cm}$ arc filling defect of the lower thoracic esophagus was revealed in esophagography. Chest Computed Tomography (CT) revealed uneven eccentric thickness on the lower esophageal wall and lumen stenosis of the esophagus. The abdominal CT showed suspicious thickness on the esophagocardiac wall, lymph node enlargement in hepatogastric ligament and splenogastric ligament. Besides, the abdominal CT also revealed a heterogeneous mass measuring $10 \mathrm{~cm} \times 10 \mathrm{~cm} \times 8 \mathrm{~cm}$ in the inferior-left abdomen, the region of the small bowel (Figure1C). Upper gastrointestinal endoscopic examination showed sporadic leukoplakia at $28-35 \mathrm{~cm}$ from the incisor, and a nodular mass with ulcer and fragile mucosa was revealed between $35-37 \mathrm{~cm}$ from the incisor (Figure1A). Another nodular mass, with ulcer and irregularities, was found at the gastric angle (Figure1B). Biopsies from the first lesion turned out to be mild dysplasia squamous epithelial hyperplasia, and the second one was squamous cell carcinoma. Tissue from the gastric angle revealed partial glandular epithelium moderately-to-poorly dysplasia, focal cancerous, mucosal carcinogenesis. Overall, the preoperative diagnosis was esophageal cancer with gastric angle cancer and occupations of abdominal cavity.

Initially, a total gastrectomy and subtotal esophagectomy were planned. Patient, firstly underwent exploration of the peritoneal cavity. A huge tumor measuring $11.5 \mathrm{~cm} \times 8.5 \mathrm{~cm} \times 7 \mathrm{~cm}$ was located in the jejunal wall (Figure 2a), approximately $60 \mathrm{~cm}$ distal to the ligament of Treitz. Frozen biopsy of the tumor turned out to be a fusicellular tumor, which tended to be GIST. Thereafter, total gastrectomy and segmental jejunectomy with regional lymph node dissection was performed. A pedicled-jejunal-interposition of adequate length was prepared for esophageal reconstruction and a Roux-en-Y procedure was performed to restore intestinal continuity. Then, esophagectomy with radical mediastinal lymph node dissection were performed through the left fifth intercostal thoracotomy. The jejunum was lifted through the retrocolic route into chest, and an end to side hand sewn intrathoracic esophagojejunal anastomosis was completed (Figure 2b). Total time of operation was 276 minutes and the estimated blood loss was about $420 \mathrm{~mL}$. 


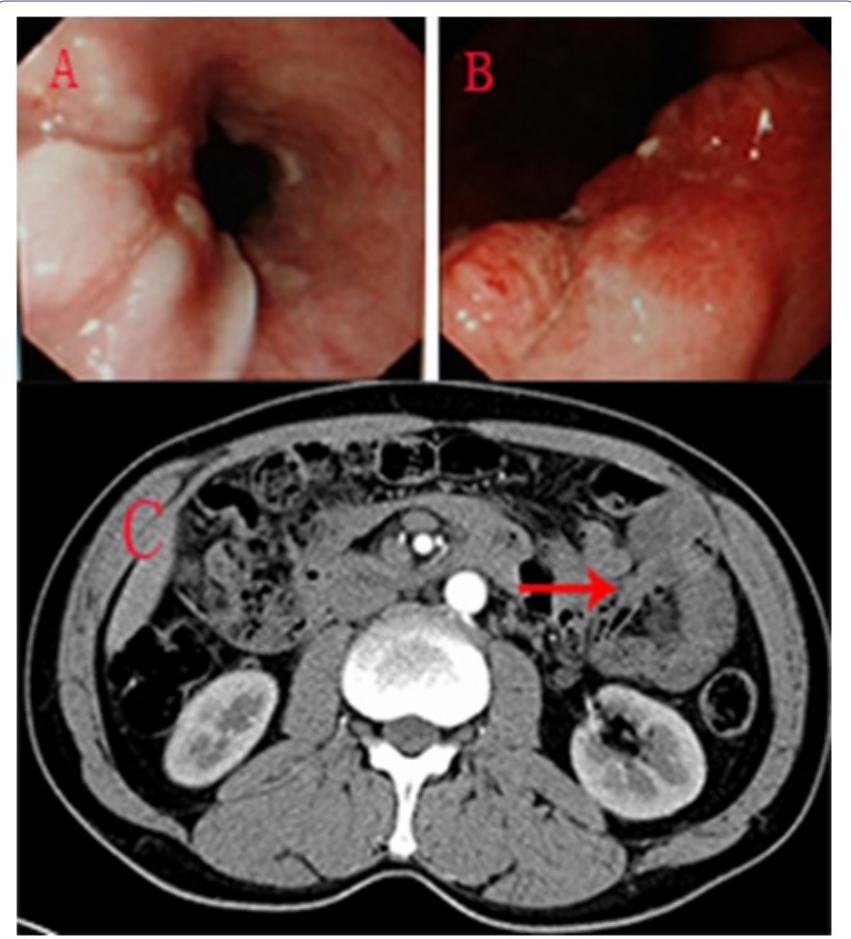

Figure 1: A - Gastrointestinal endoscopy showed a nodular mass with ulcer and fragile mucosa on the anterior wall of the low thoracic esophagus; B - A nodular mass, with ulcer and irregularities on the gastric angle; and C- Abdominal CT scan showed heterogeneous mass (red arrow) in the inferior-left abdomen.
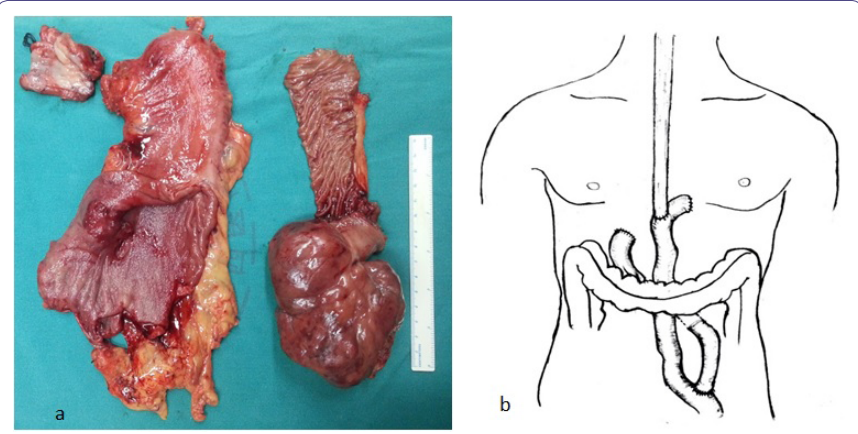

Figure 2: (a) All the resected tumor : esophagus, stomach and jejunum; (b) Alimentary tract restoration using jejunum with vascular pedicle via the retrocolic route into chest and an end to side hand sewn intrathoracic esophagojejunal anastomosis were performed.

Histopathologic examination of the esophageal tumor was moderately differentiated squamous cell carcinoma involving adventitia (Figure 3a). Three groups of lymph nodes with squamous cell carcinoma metastasis were detected in lower paraesophageal lymph nodes $(1 / 1)$, left tracheobronchial nodes $(2 / 2)$ and paracardial nodes (3/8). The gastric angle one was diagnosed as moderately-to-poorly differentiated adenocarcinoma (Tubular adenocarcinoma and Signet ring cell carcinoma), which invaded subserosal connective tissue without invasion of visceral peritoneum or adjacent structures (Figure $3 \mathrm{~b}$ ), with regional lymph nodes metastasis of adenocarcinoma, including left gastric nodes $(1 / 1)$, celiac nodes $(1 / 1)$, the greater omentum lymph nodes (1/2), and lymph nodes of lesser curvature stomach $(2 / 2)$. The gastric tumor was positive for Her-2 (2+). The jejunal tumor was a fusicellular tumor, which was positive for CD117, DOG-1, SMA (Figure 3c), and its mitotic rate was less than 5 per 50 HPF. Considering the size of the tumor (more than $10 \mathrm{~cm}$ in greatest dimension), final diagnosis of the jejunal tumor was small intestinal GIST, without any regional lymph node metastasis or distant metastasis. Overall, based on the seventh edition of the American Joint Committee on Cancer (AJCC) staging manual, the postoperative diagnosis was lower esophageal squamous cell carcinoma (pT3N2M0, G2, Stage B), gastric angle adenocarcinoma (pT3N2M0) and Gastrointestinal Stromal Tumor (GIST) in the jejunum (pT3N0M0, Low, Stage II).

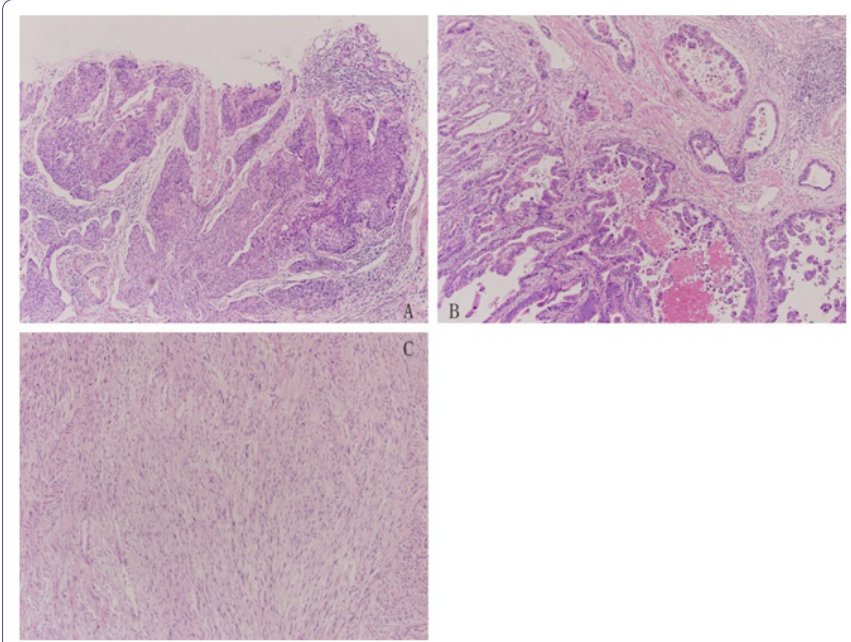

Figure 3: (a) Pathological specimen from the esophageal tumor showed a moderately differentiated squamous cell carcinoma (HE $\times 100)$; (b) Pathological specimen from the gastric tumor revealed a moderately-to-poorly differentiated adenocarcinoma (HE×100); and (c) Pathological specimen from the jejunal tumor showed a fusicellular tumor ((HE×100)).

Postoperative course was uneventful. He started a liquid diet after 5 day's parenteral nutrition with integrity of anastomosis confirmed by the esophagography. Without any major postoperative complications, he was discharged 15 days after the operation. Three months after operation, without anastomotic stenosis, which was confirmed by barium esophagography (Figure 4). Considering his continuous low white blood cell levels, physical and economic conditions, he didn't receive radiotherapy and started to receive SOX adjuvant chemotherapy for six cycles with cisplatin and S-1 (an oral fluoropyrimidine produced by combining tegafur, a prodrug of 5-FU, with 5-chloro-2, 4-dihy-droxypyridine and potassium oxonate) [8]. After 12 months of follow-up, the patient is still alive and under regular follow-up.

\section{Discussion}

According to the concept of 'field cancerization' [9], the aerodigestive tract turns out to be a place where multiple primary cancers occur mostly. And, it is reported that gastric cancer is one of the most common primary cancer comes out with esophageal cancer synchronously [10-14]. However, the rare event is that primary cancers of esophagus and stomach associated with the third organ, especially the third one is GIST of the jejunum, arise synchronously.

Review of the literature revealed only two cases with primaries involving esophagus, stomach and jejunum, and one of them was autopsy case [1-2]. Moreover, none of these cases was a synchronous one or included a third cancer of jejunal GIST. To our knowledge, this is the first reported case of resected triple primary cancers involving the esophagus, stomach and jejunum synchronously.

GISTs are the most common non-epithelial tumors of the gastrointestinal tract and mostly treated by surgery. About ninety 
Citation: Wei-Peng Hu, Yu-Shang Yang, Jun-He Gou, Yuan Y, Wen-Ping Wang, et al. (2016) Synchronous Triple Primary Cancer of the Esophagus, Stomach and Jejunum: A Case Report and Review of Literature. J Clin Stud Med Case Rep 3: 029.

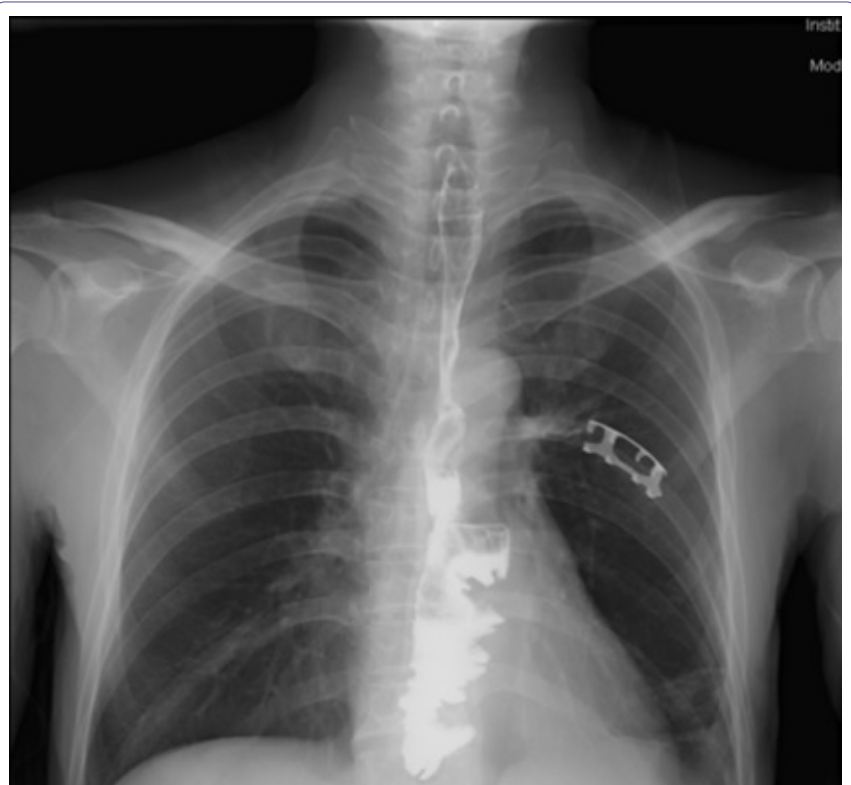

Figure 4: Barium esophagography three months after the operation.

percent of GISTs were detected clinically due to symptoms (69\%) or were incidental findings at surgery (21\%); the remaining $10 \%$ of GISTs were found at autopsy [15]. In this case, the jejunal GIST was detected incidentally without showing any related signs. Thus, given the size and location of the abdominal mass, we chose abbreviated laparotomy to clarify origin of tumor. The reconstruction of the digestive tract determines the success of synchronous esophageal and gastric cancer. Considering that the jejunum is a practical alternative to the colon when the stomach is not available [16], we chose an apedicled-jejunal-interposition for the reconstruction of esophagus. There were no severe complication occurred after the operation, and most of all, the nutritional status was maintained. Therefore, we concluded that this surgical procedure was acceptable.

On his first admission to our department, he had been suffering from durative dysphagia for over 1 year, resulting in his bad physical and nutritional status. Thus, we concluded that he couldn't tolerate neoadjuvant chemotherapy before surgery, although he might have the indication. Combination of chemotherapy and radiation therapy have been proven to be of great contribution to overall survival rate of esophageal squamous cell carcinoma, especially for the locoregionally advanced one [17-18]. In spite of the fact that chemotherapy protocols with imatinib can apparently improve recurrence-free survival rate and overall survival of GIST patients [19], the cost for the whole course, to a certain extent, is too expensive for this family to afford. His white blood cell levels was persistently under $2.9 \times 10^{9} / \mathrm{L}$ and physical status was still poor after the surgery. Given that, he finally received SOX adjuvant chemotherapy without radiotherapy or imatinib.

In general, the combination of the three different neoplasms (esophageal squamous cell carcinoma, gastric angle adenocarcinoma and GIST in the jejunum) in one patient synchronously, to the best of our knowledge, has never been reported before.

\section{References}

1. Kato Y, Tsuyuki A, Kikuchi K, Fujishiro Y, Tanabe M, et al. (2008) Primary jejunal adenocarcinoma as part of multiple primary cancers of the digestive tract. J Gastroenterol Hepatol 23: 673-677.
2. The Japanese Society of Pathology (2000) Annual of the Pathological Autopsy Cases in Japan. Nihon Byori Bouken Shuho Kankokai, Tokyo, Japan.

3. Xie S, Huang J, Kang G, Fan G, Wang W (2013) Surgical treatment of synchronous gastric and esophageal carcinoma: case report and review of literature. Thorac Cardiovasc Surg Rep 2: 35-37.

4. Fukaya M, Abe T, Yokoyama Y, Itatsu K, Nagino M (2014) Two-stage operation for synchronous triple primary cancer of the esophagus, stomach, and ampulla of Vater: report of a case. Surg Today 44: 967-971.

5. Anastassios V Koutsopoulos, Konstantina I Dambaki, George Datseris, Elpida Giannikaki, Marios Froudarakis, et al. (2005) A novel combination of multiple primary carcinomas: Urinary bladder transitional cell carcinoma, prostate adenocarcinoma and small cell lung carcinoma- report of a case and review of the literature. World J Surg Oncol 3: 51.

6. Munker R, Hiller E, Melnyk A, Gutjahr P (1996) Second malignancies. Int J Oncol 9: 763-776.

7. Watanabe S, Kodama T, Shimosato $\mathrm{Y}$, Arimoto H, Sugimura T, et al. (1984) Multiple primary cancers in 5,456 autopsy cases in the National Cancer Center of Japan. J Natl Cancer Inst 72: 1021-1027.

8. Takii Y, Yamazaki T, Okada T, Tani T, Funakoshi K, et al. (2013) Phase I/II trial of irinotecan and S-1 combination chemotherapy as a second-line treatment for advanced colorectal cancer. Chemotherapy 59: 338-343.

9. Strong MS, Incze J, Vaughan CW (1984) Field cancerization in the aerodigestive tract--its etiology, manifestation, and significance. J Otolaryngol 13: $1-6$.

10. Kumagai Y, Kawano T, Nakajima Y, Nagai K, Inoue H, et al. (2001) Multiple primary cancers associated with esophageal carcinoma. Surg Today 31: $872-$ 876.

11. Natsugoe $S$, Matsumoto $M$, Okumura $H$, Ishigami $S$, Uenosono $Y$, et al. (2005) Multiple primary carcinomas with esophageal squamous cell cancer: clinicopathologic outcome. World J Surg 29: 46-49.

12. Poon RT, Law SY, Chu KM, Branicki FJ, Wong J (1998) Multiple primary cancers in esophageal squamous cell carcinoma: incidence and implications. Ann Thorac Surg 65: 1529-1534.

13. Vrabec DP (1979) Multiple primary malignancies of the upper aerodigestive system. Ann Otol Rhinol Laryngol 88: 846-854.

14. Shons AR, McQuarrie DG (1985) Multiple primary epidermoid carcinomas of the upper aerodigestive tract. Arch Surg 120: 1007-1009.

15. Nilsson B, Bümming $P$, Meis-Kindblom JM, Odén A, Dortok A, et al. (2005) Gastrointestinal stromal tumors: the incidence, prevalence, clinical course, and prognostication in the preimatinib mesylate era--a population-based study in western Sweden. Cancer 103: 821-829.

16. Blackmon SH, Correa AM, Skoracki R, Chevray PM, Kim MP, et al. (2012) Supercharged pedicled jejunal interposition for esophageal replacement: a 10-year experience. Ann Thorac Surg 94: 1104-1111.

17. Herskovic A, Martz K, Al-Sarraf M, Leichman L, Brindle J, et al. (1992) Combined chemotherapy and radiotherapy compared with radiotherapy alone in patients with cancer of the esophagus. N Engl J Med 326: 1593-1598.

18. Ruppert BN, Watkins JM, Shirai K, Wahlquist AE, Garrett-Mayer E, et al. (2010) Cisplatin//rinotecan versus carboplatin/paclitaxel as definitive chemoradiotherapy for locoregionally advanced esophageal cancer. Am J Clin Oncol 33: 346-352.

19. Joensuu H, Eriksson M, Sundby Hall K, Hartmann JT, Pink D, et al. (2012) One vs three years of adjuvant imatinib for operable gastrointestinal stromal tumor: a randomized trial. JAMA 307: 1265-1272. 